133 EFFECT OF INTRAVENOUS TMMUNOGIOBULTN /IVIG/ TREATAgnes Kalmár, Imre Szabó, László Meródi University of Wedical School of Debrecen, Department of Pediatrics, Debrecen, Hungary

In this study the effect of IVIG treatment on the activation of $C$ was investigated. The patients' group consisted of li infants with bacterial sepsis. The conventional antimicrobial therapy in these patients was supplemented with acid / $\mathrm{pH} 4 /$ treated immunoglobulin for three days / daily dose $30 \mathrm{mg} / \mathrm{kg} /$. The possible effect of the IVIG treatment on the activation of $\mathrm{C}$ was studied by measuring the CH5O and AP5O activity as well as concentration of $\mathrm{C} 3$ and $\mathrm{C} 3 \mathrm{~d} / \mathrm{C} 3 \mathrm{dg}$ before and soon after the first dose of noblobulin was given and on the day after the three day treatment /Table/.

$\begin{array}{llll} & \text { Before treatment } & \text { After the first dose After treatmeni } \\ \mathrm{CH} 50 / \mathrm{ml} & 37,5 & - & 36,2 \\ \mathrm{AP} 50 / \mathrm{ml} & 25,8 & - & 27,3 \\ \mathrm{C} 3 \mathrm{~g} / \mathrm{I} & 0,8 & - & 0,86 \\ \mathrm{C} 3 \mathrm{~d} / \mathrm{C} 3 \mathrm{dg}^{+} & 30,5 & 15,5 & 19,5\end{array}$

texpressed as the percentage of

Taken together these results indicate no significant alteration

in $\mathrm{C}$ activation during IVIG treatment of septic infants.

\title{
Author Index to Abstracts
}

Numbers cited refer to abstract numbers, except plenary lectures which are cited by page number.

Aass $\mathrm{H}, 120$

Acolet $\mathrm{D}, 49$

Acsádi G, 12

Agati $G, 102$

Agostoni C, 59

Agsteribbe E, 35

Alagille $\mathrm{D}, \mathrm{T} 2$

Albani M, 37

Alison ME, 38

Andersen O, 131

Andersson S, 25

Argiolas L, 43

Arleth S, 128

Assenbaum E, 7

Aviram M, 119

Avison M, 116

Azzopardi D, 41

Baarsma R, 94

Bachman C, 116

Baerts W, 5

Balata A, 43

Bamberger U, 4

Bamford O, 5

Baraldi E, 127

Barba BD, 132

Barginda M, 53

Barks J, 100

Bartmann P, 3, 4, 87

Batchiulis V, 60

Baudin J, 39, 4

Bauer K, 27, 95

Beetz R, 73, 85

Beisiegel U, 11

Bellu R, 59

Bengtsson A, 6

Bentele KHP, 37

Benz R, 93

Ben-Zvi Z, 8

Berebi A, 63

Berger R, 35, 94, 119

Bernardini I, 30

Bertele RM, 128

Besana R, 59

Bjørnstad PG, 64

Blackledge MJ, 19

Blanco CE, 5, 16, 113

Böck P, 91

Böhler T, 26
Böhles HJ, 99

Bolas NM, 20

Bossi E, 67

Böswald W, 99

Böwing B, 99

Bradford BC, 40

Brand R, 50

Brandtzaeg P, 130

Bratlid D, 45, 46, 47, 97

Brenner RE, 117

Brubacher G, 80

Bryan EM, 38, 54, 55

Bucher HU, 52

Burgio RG, 62

Büttner HJ, 85

Cady EB, 41

Calame A, 44

Cantarutti F, 132

Chapman T, 94

Chapman TE, 76

Chen V, 5

Chiarelli F, 77

Chiesa C, 86

Cianfrano V, 86

Clark KGA, 82

Clemons GK, 84

Cole CB, 101

Colombo J, 116

Corbeel L, 34

Corchia C, 43

Cossack ZT, 89

Cotes MP, 82

Cotes PM, 81

Cowan F, 18

Da Dalt L, 127

Dahlin I, 104

Dalinghaus M, 124, 125, 126

de Boer WJ, 126

Degraeuwe PLJ, 113

Del Principe D, 23, 86

Delpy DT, 41

De Matteis W, 23

Dhondt JL, 32

Di Bari A, 122

Di Fabio S, 98, 122

Di Giulio S, 23
Disse B, 3

Dobrzańska A, 65

Domizio $\mathrm{S}, 98$

Donzelli F, 127

Donzelli GP, 102

Dörner K, 58

Drackenberg K, 70

Duc G, 52

Edberg KE, 2

Edwards D, 39

Ekström-Jodal B, 2

Elliman AD, 38, 54, 55

Elliman AM, 54,55

Eriksson BO, 31

Ermisch J, 92

Fanconi S, 52

Farriaux JP, 32

Fawer C, 44

Fearne JM, 55

Fetter WPF, 51

Finazzi-Agrò $\mathrm{A}, 23$

Forteleoni $\mathrm{G}, 43$

Friberg LG, 6

Fulconis F, 75

Füllenkemper F, 13

Fuller R, 101

Fusi F, 102

Gahl W, 30

Galluzzo C, 59

Galvan P, 102

Gangitano ES, 28

Gardiner RM, 20

Gaustad P, 130

Gehrhardt B, 110

Geiger R, 119

Geller-Bernstein C, 63

Gerbault L, 111

Gerboni S, 122

Gerding AM, 124, 125, 126

Gieles $\mathrm{P}, 1$

Giordani M, 23

Giovannini M, 59

Gitzelmann R, 14

Glatzl-Hawlik M-A, 68

Gmyrek D, 110
Goßla R, 72

Gorodischer R, 8

Gortner L, 3, 4, 87

Gotsauner F, 91

Gratama JWC, 124, 125, 126

Grau J, 7

Groenendaal F, 51

Grögaard JB, 112

Gutteberg TJ, 131

Hågå $\mathrm{P}, 81$

Haira A, 100

Hall $\mathrm{K}, 70$

Hall MA, 96, 101

Hallman M, 2, 10, 25

Hall SM, T1

Halvorsen S, 36, 84, 130

Hammarlund K, 69

Hansen TWR, 21, 45, 46, 47, 97

Hanson MA, 113

Harms KH, 128

Hartmann W, 72

Harvey DR, 38

Hascoet J-M, 112

Hawkings R, 5

Hayte JM, 32

Hazell JWP, 40

Heersema DJ, 51

Heideman M, 6

Heimler R, 109

Held A, 56

Hellebostad M, 81

Heller-Schöch G, 56

Hennessy VS, 114

Henrichs I, 93

Hermetter A, 57

Herschkowitz N, 67, 116

Herzog KH, 99

Heymans HSA, 113

Hjalmarson $\mathrm{O}, 2$

Holliday DJ, 114

Holmes RD, 100

Hope PL, 19

Höyer S, 31

Hübner C, 11

Isken VHA, 123

Ives NK, 19, 20 
Jaeken $\mathrm{J}, 34$

Jonitz W, 7

Jorch $G, 53,103$

Jørgensen T, 131

Juberg RC, 114

Kalmár Ả, 133

Kassur-Siemieńska B, 65

Kellner M, 93

Kelly FJ, 96

Kerner J, 12

Kierulf $P, 130$

Koers JH, 124, 125, 126

Kohler E, 67

Kohlschütter A, 11

Koletzko B, 74

Korinthenberg R, 13

Körner K, 88

Kuipers JRG, 124, 125, 126

Kumar P, 113

Kvittingen EA, 36

Kwant G, 126

Lachmann B, 108

Lafeber HN, 76

Lagercrantz H, 104

Lakatos J, 12

Langslet A, 120

LaPenna G, 77

Leitz R, 90

Leonhardt A, 123

Licht A, 63

Linderkamp O, 26, 27, 123

Lindner W, 29, 121

Lindstedt S, 31

Lloyd B, 39

Lorini R, 62

Lund HT, 115

Lüthy C, 60

Maertzdorf WJ, 16

Manfra T, 77

Mannhardt W, 73, 85

Maródi L, 133

Martini A, 62

Matsuo M, 107

Matsuo T, 107

Meberg A, 42, 61

Mehls O, 83

Meister JJ, 44

Melegh B, 12

Melino G, 23

Mellgren G, 6

Menichelli A, 23

Meuzelaar JJ, 124, 125

Michalk DV, 90

Midulla M, 86

Milewska-Bobula B, 65

Miller E, 128

Minoli I, 75

Mjøs OD, 48

Molnár D, 79

Moncha M, 124

Montini G, 82

Morgese G, 77

Moro G, 75

Morris MC, 82

Moses SW, 119

Motoike T, 107

Müller J, 74
Müller PK, 117

Müller-Wiefel DE, 83

Muraro MA, 127

Murer L, 132

Nakamura H, 107

Nanni F, 86

Nichetti C, 127

Nicolai T, 128

Nijenhuis F, 125

Nolte $S, 7$

Nordin I, 31

Norstein J, 24

Novotny E, 116

Oetliker O, 60

Oetomo SB, 1, 108

Okken A, 1, 94, 108

Oldfors A, 31

Oldigs H-D, 58, 88

Ortega E, 63

Ortisi MT, 59

Orzalesi M, 43

Osnes J-B, 106, 120

Øyasæter S, 46, 97

Pacifico L, 86

Pecht I, 63

Pecoraro AR, 98, 122

Pelegano J, 109

Persson B, 70

Petroff $\mathrm{O}, 116$

Philipps AF, 70

Piatkowska E, 9

Pietrzyk JJ, 9, 129

Pitkänen $\mathrm{O}, 25$

Plenk H, 91

Pohlandt F, 3, 4, 87

Pórszász J, 79

Poulsen JP, 47

Prichard J, 116

Quartulli L, 98, 122

Rabe H, 53, 103

Radda GK, 19

Raivio KO, 22, 66

Rajagopalan B, 19

Ramenghi LA, 98, 122

Rauch J, 90

Ravelli A, 62

Reichelt KL, 64

Reijngoud D-J, 1, 94

Reinhold P, 103

Renlund M, 30

Resta V, 122

Reynolds EOR, 39, 40, 41

Riegel K, 29, 121

Riesenfeld T, 69

Rigden SPA, 82

Ringstad J, 48

Riva E, 59

Rizzo E, 86

Rodgers W, 96

Røkke O, 131

Rolles CJ, 101

Rossier MT, 44

Rowecka-Trzebicka K, 65

Rubaltelli FF, 127, 132
Ruggiero I, 129

Ruth V, 22

Ruys JH, 50

Sabatino G, 98, 122

Sabel K-G, 31

Sagvolden T, 45

Saia SO, 132

Sandberg K, 2

Sander G, 56

Sanderud J, 24

Sándor A, 12

Sanengen $T, 84$

Sanna MC, 43

Sano K, 107

Sara V, 70

Sasidharan P, 109

Sauer PJJ, 76

Saugier P, 111

Saugstad OD, 24, 47

Savini I, 23

Schadewaldt P, 15

Schaller P, 110

Schaub J, 58, 88

Schaumberger M, 121

Schiander IG, 106

Schindler M, 29

Schmiereck S, 33

Schöch G, 56

Schofer $O, 73,85$

Schönau E, 99

Schoots C, 108

Schulte FJ, 37

Schulze A, 110

Schutgens RBH, 118

Schwehm P, 83

Scigalla $P, 83$

Sedin G, 69

Selstam U, 112

Semb G, 120

Seppälä R, 30

Serra C, 43

Shulman R, 116

Sievers E, 58, 88

Silberberg A, 2

Simbruner G, 68, 91

Sipilä I, 10

Skomedal T, 106, 120

Slaaf DW, 16

Sleath K, 49

Smith S, 101

Smit PC, 119

Snyder JR, 28

Sørensen E, 130

Søvik O, 36

Stadler A, 26

Steen-Johnsen J, 36

Steinmann B, 14

Stephani U, 71

Stewart A, 39, 40

Stewart AL, 41

Stinson RA, 91

Stiris T, 46, 97

Sulkers EJ, 76

Sundell HW, 112

Sutter A, 71

Sutton P, 19

Szabó I, 133

Szydlowska T, 129
Tangelder GJ, 16

Teller WM, 92, 93, 117

Thoresen M, 17, 104, 105

Tietze F, 30

Tomlinson LL, 82

Toplak H, 57, 60

Topp H, 56

Tordet C, 111

Townsend J, 39

Tranebjærg L, 115

Turck D, 32

Ullrich $\mathrm{K}, 13,33$

Uracz W, 129

van Asselt WA, 94

van Bel F, 28

van den Anker JN, 76

van den Berg ME, 126

van der Berg I, 35

van Faassen $H, 35$

van Hof-van Duin J, 51

van Zeben der Aa DM, 50

Verloove-Vanhorick SP, 50

Versmold HT, 27, 29, 95, 121

Verwey RA, 50

Vettenranta K, 66

Vetter U, 72, 92, 117

von Figura K, 33

von Känel J, 14

Vozzi A, 132

Walaas SI, 21

Walker JA, 54

Walther FJ, 28

Walti H, 111

Wanders RJA, 113

Wanner R, 99

Weller E, 3

Wendel U, 15

Weninger M, 91

Westre B, 45

Whitelaw A, 17, 18, 49, 105

Widhalm K, 78, 80

Widness JA, 84

Wiehler U, 11

Wiemer E, 35

Wiesmann U, 60

Wiesmann UN, 14, 57

Wildevuur C, 1

Willekens $\mathrm{H}, 34$

Williams BA, 113

Wilson GN, 100

Wittich U, 93

Wörsdörfer A, 92

Wörsdorfer O, 117

Woyke H, 103

Wyatt JS, 41

Yaari A, 8

Ytrehus K, 48

Zabel B, 73

Zanaboni D, 62

Zembala M, 129

Zepp F, 73, 85

Zijlstra WG, 126

Zilow E, 26

Zimmermann A, 71

Zwiauer K, 78, 80) 